\title{
Analysis of antithrombotic therapy in elderly patients with atrial fibrillation
}

\author{
W.Q. Gao, Y.T. Guo, J.L. Ma, P. Zhu and Y.T. Wang \\ First Geriatric Cardiology Division of South Building, \\ Chinese PLA General Hospital, Beijing, China \\ Corresponding author: W. Gao \\ E-mail: wenqiangaocn@126.com
}

Genet. Mol. Res. 13 (1): 736-743 (2014)

Received May 17, 2013

Accepted December 7, 2013

Published January 29, 2014

DOI http://dx.doi.org/10.4238/2014.January.29.4

\begin{abstract}
This study aimed to analyze the impact factors and outcome of antithrombotic therapy in elderly patients over 65 years old that suffered from atrial fibrillation (AF). A total of 256 elderly patients with $\mathrm{AF}$ over 65 years old were divided into 3 groups: 6574 years old $(\mathrm{N}=86), 75-84$ years old $(\mathrm{N}=122)$, and over 85 years old $(\mathrm{N}=48)$. The clinical characteristics, antithrombotic therapy, and its related impact factors were retrospectively analyzed. Of all patients, 187 received antithrombotic therapy. In the 65-74 yearold group, 78 patients received antiplatelet treatment $(90.7 \%)$ and 5 patients received anticoagulation treatment (5.8\%). In the $75-84$ year-old group, 76 patients received antiplatelet treatment $(62.3 \%)$ and 14 patients received anticoagulation treatment $(11.5 \%)$. In the group of over 85 year-olds, 33 patients received antiplatelet therapy $(68.8 \%)$ and 4 patients received anticoagulation treatment $(8.3 \%)$. Eleven patients had deep vein thrombosis and atrial thrombosis during antiplatelet therapy (5.9\%), 5 patients had gastrointestinal hemorrhage after antiplatelet therapy (2.7\%), 2 patients had gastrointestinal bleeding, and 3 patients had brain hemorrhage after anticoagulation treatment $(21.7 \%)$. Suboptimal antithrombotic therapy was observed
\end{abstract}


in the elderly patients with AF, partly owing to the risks of both thromboembolism and bleeding.

Key words: Antithrombotic therapy; Atrial fibrillation; Elderly patients

\section{INTRODUCTION}

Atrial fibrillation (AF) is the most common arrhythmia observed in clinical practice (Crandall et al., 2009). The incidence of AF increases with age, and for elderly patients aged over 80 years, it can reach up to $10 \%$ (Go et al., 2001; Freestone and Lip, 2003). Ischemic stroke and other thromboembolic events are the major AF-related complications (Spence, 2009). Thromboembolic events could occur at least once in $35 \%$ of patients with AF. Moreover, recurrent stroke in patients who had a stroke or transient ischemic events are widely common, with the incidence reaching up to $12 \%$. Old age is not only a risk factor for developing AF, but is also an independent risk factor for thromboembolic events, including ischemic stroke (Lin et al., 1996; Marini et al., 2005; Böhm et al., 2009). Therefore, elderly patients can benefit more from antithrombotic therapy. In recent years, the management of thrombosis prevention in AF patients has shown continuous development. The European Society of Cardiology (ESC) published related guidelines in 2010 (hereafter referred to as the ESC2010 guidelines) (Camm et al., 2010). Subsequently, the American College of Cardiology Foundation (ACCF)/American Heart Association (AHA)/Heart Rhythm Society (HRS) updated their guidelines (hereafter referred to as ACCF/AHA/HRS 2011 guidelines) (Fuster et al., 2011; Wann et al., 2011a,b), and improved anticoagulant treatment of AF. Based on CHA2DS2-VSAc scoring, the ESC guidelines for the stratification of stroke risk in patients with $\mathrm{AF}$ in 2010 stated that effective anticoagulation treatment was recommended for almost all elderly patients with AF. However, because of complex pathophysiological changes in elderly patients, there are still gaps between the ESC guide and antithrombotic therapy for AF in China. This study aimed to evaluate the current state of antithrombotic therapy in elderly patients with AF in everyday clinical practice in China, analyze the factors influencing the treatment, and provide clinical advice for the standardization of antithrombotic therapy for elderly patients.

\section{MATERIAL AND METHODS}

\section{Subjects}

A total of 256 elderly patients over 65 years old with AF admitted to our hospital in the past 3 years were recruited for the study, including 249 males and 7 females. The average age of all patients was $80.6 \pm 7.2$ years (range: $65-98$ years). The primary diseases of the patients were as follows: 229 patients had coronary atherosclerotic heart disease, 225 patients had hypertensive heart disease, 7 patients had dilated cardiomyopathy, 54 patients had pulmonary heart disease, and 3 patients had hyperthyroid heart disease. This study was conducted in accordance with the declaration of Helsinki and with approval from the Ethics Committee of the Chinese PLA General Hospital. Written informed consent was obtained from all participants. 
According to the suggestions of the working group of arrhythmia of the European Society for Cardiovascular Disease (WGA-ESC) and the North American Society of Pacing and Electrophysiology (NASPE), the classification of AF was as follows: 1) in paroxysmal $\mathrm{AF}$, the mean duration of AF is less than 7 days, and generally $<48 \mathrm{~h}$, and is mostly selflimiting; 2) in persistent $\mathrm{AF}$, the duration of $\mathrm{AF}$ is more than 7 days, or less than 7 days but patients need drugs or electrical cardioversion; 3) in permanent $\mathrm{AF}$, AF cardioversion fails or cardioversion is no longer attempted (Lévy et al., 2003). The AF of the 256 patients was confirmed by electrocardiogram, of which 156 cases had paroxysmal AF, 64 cases had persistent AF, and 36 cases had permanent AF. The AF course ranges from 8 months to 36 years, and the average course is $15.8 \pm 6.2$ years. Accessory examinations were conducted to determine whether the left atrial diameter was $38 \pm 3 \mathrm{~mm}$ under cardiac ultrasonography.

\section{Grouping}

All patients were divided into three groups by age: the 65-74 year-old group (86 cases), the 75-84 year-old group (122 cases), and the over 85 year-old group ( 48 cases).

\section{Treatment}

A total of 243 patients received drug therapy, including Metoprolol, Cordarone, and Propafenone, to control the ventricular rate. Aspirin and clopidogrel were used for antiplatelet therapy, and warfarin was used as anticoagulation therapy. Thirteen patients received pacemaker (VVI, Medtronic) treatment. The indications for VVI pacemaker therapy were persistent $\mathrm{AF}$ with a slow ventricular rate and an atrioventricular conduction blockage.

\section{Statistical analysis}

All parameters are reported as means \pm standard deviation. The discrete variables are expressed as percentages. The SPSS18.0 statistical software was adopted for data analysis, and comparisons between groups were conducted using the chi-squared test. $\mathrm{P}<0.05$ indicated that differences were considered to be statistically significant.

\section{RESULTS}

\section{Antithrombotic therapy}

Of all patients, 210 received antithrombotic treatment (82.0\%) and $46(18.0 \%)$ did not. Antithrombotic aspirin was used in 75 patients, clopidogrel was used in 78 patients, 34 patients were treated with both aspirin and clopidogrel, and 23 patients received warfarin. In patients aged $65-74$ years old, $90.7 \%$ of patients received antiplatelet therapy, and $5.8 \%$ received anticoagulant therapy. In patients aged $75-84$ years, $62.3 \%$ of patients received antiplatelet therapy and $11.5 \%$ received anticoagulant therapy. In patients over 85 years old, $68.8 \%$ of patients received antiplatelet therapy and $8.3 \%$ received anticoagulation therapy. The use of antithrombotic therapy with respect to the different age groups is shown in Table 1. 
Table 1. Antithrombotic therapy in relation to different age.

\begin{tabular}{lccc}
\hline Group & Number of cases & Antiplatelet therapy $(\%)$ & Anticoagulant therapy $(\%)$ \\
\hline $65-74$ & 86 & $78(90.7 \%)$ & $5(5.8 \%)$ \\
$75-84$ & 122 & $76(62.3 \%)^{*}$ & $14(11.5 \%)^{*}$ \\
Over 85 & 48 & $33(68.8 \%)^{*}$ & $4(8.3 \%)^{*}$ \\
\hline
\end{tabular}

Compared with the 65-74 year-old group, $* \mathrm{P}<0.05$.

\section{Comorbidities in elderly patients}

Fifty patients had tumors (20.3\%), 9 cases of which were seen in the $65-74$ year-old group, 28 were in the 75-84 year-old group, and 13 were in the over 85 year-old group. The tumors were observed in several different systems, including meningiomas, nasopharyngeal squamous cell carcinoma, parathyroid adenoma, lung cancer, stromal tumor in the stomach, gastric cancer, cardia carcinoma, hepatocellular carcinoma, carcinoma of the sigmoid colon, renal clear cell carcinoma, renal papillary carcinoma, bladder cancer, prostate cancer, and chronic lymphocytic leukemia (Table 2).

Table 2. Patients who had tumor in different age.
\begin{tabular}{lc}
\hline Group & No. of cases \\
\hline $65-74$ & 9 \\
$75-84$ & 28 \\
Over 85 & 13 \\
\hline
\end{tabular}

Of all patients, 42 had prior bleeding or diseases of bleeding tendency before antithrombotic treatment, including brain hemorrhage, hemorrhagic gastritis, duodenal bulb ulcer, renal anemia, thrombocytopenia, and so on. Fifty-two patients had thrombosis or embolism disease tendencies before antithrombotic therapy, including atrial thrombosis, deep venous thrombosis, insufficient blood supply of vertebral basilar artery, cerebral lacunar infarction, peripheral vascular embolization, and so on. Twenty-three of these patients had two kinds of the diseases mentioned above.

\section{Effects of antithrombotic therapy}

Eleven patients had atrial thrombosis and deep vein thrombosis during antiplatelet therapy (5.9\%); 1 patient was 65-74 years old, 7 patients were 75-84 years old, and 3 patients were over 85 years old. There was no new onset atrial thrombosis or deep vein thrombosis events in patients who received anticoagulant therapy (Table 3).

\begin{tabular}{lc} 
Table 3. Patients who had thrombosis in different age. \\
\hline Group & No. of cases \\
\hline $65-74$ & 1 \\
$75-84$ & 7 \\
Over 85 & 3 \\
\hline
\end{tabular}




\section{Safety of antithrombotic therapy}

Five patients had gastrointestinal hemorrhages during antiplatelet therapy $(2.7 \%)$. Two patients had gastrointestinal hemorrhage and 3 patients had brain hemorrhages during anticoagulant therapy $(21.7 \%)$.

\section{DISCUSSION}

$\mathrm{AF}$ is often accompanied by many diseases in elderly patients. In this study, we observed that most elderly patients with AF had comorbidities such as coronary heart disease, hypertension, and diabetes. We ranked the risk of stroke related to AF according to the CHADS2 score. Patients with a low risk of a thromboembolic event may benefit from aspirin treatment. The combination of aspirin and clopidogrel or warfarin should be considered for patients with intermediate and high risks (Gage et al., 2001; Singer et al., 2008). Most of the elderly patients with AF have an intermediate or high risk of stroke, and therefore the combination of antiplatelet or anticoagulant therapy should be given. The NASPEAF random test confirmed that the incidence of AF was significantly higher in patients older than 75 years compared to that of younger patients, and combined antiplatelet and anticoagulation therapy could significantly reduce the incidence of vascular events (Pérez-Gómez et al., 2007). Lip et al. (2010) evaluated 1084 patients with AF based on the European Heart Survey with the CHA2DS2-VSAc score, confirming that the stroke incidence increased year by year as the integral age increased. Therefore, based on the CHADS2 scoring program, the new integration program filtered actual "low risk" patients while scaling down the proportion of patients with "medium risk", so that more than $90 \%$ of patients belonged to "high risk" groups. According to CHA2DS2-VSAc scoring, the ESC guidelines for the stratification of stroke risk in patients with $\mathrm{AF}$ in 2010, and recommendations that directly select antithrombotic therapy based on risk factors, almost all elderly patients with $\mathrm{AF}$ were treated with anticoagulation. The present study showed that most of the elderly patients with AF were treated with a single drug for antiplatelet therapy, and clopidogrel was most commonly used. Antiplatelet therapy was more efficient in the "younger" elderly group (65-74 year-olds) than in the older age group (over 75), which may have been due to the higher incidence of tumors, hemorrhages, and ischemia tendency diseases in the "older" elderly patients with AF. Ischemia-tendency diseases were another problem that clinicians had to face for antiplatelet therapy. Embolism occurred in $5.9 \%$ of the patients who had not achieved a sufficient protective effect from antiplatelet treatment. Only $8.9 \%$ of the patients were treated with warfarin for anticoagulation, and although embolic ischemia did not occur, bleeding occurred in $21.7 \%$ of these patients. In addition, the effect of antiplatelet therapy was not as satisfactory as that of anticoagulation therapy in elderly patients with AF. At the same time, these observations indicated that anticoagulant therapy was not adequate in elderly patients with AF; its safety was not fully monitored and the risk of bleeding was not fully confirmed. CHA2DS2-VSAc scoring is based on the European Heart Survey, and it is unknown whether any of these patients were from an Asian population. Therefore, whether CHA2DS2-VSAc scoring is suitable for the clinical diagnosis and treatment of $\mathrm{AF}$ in China needs further evaluation using a large Chinese AF cohort.

This study showed that there were comorbidities in elderly patients with AF, such as blood diseases (iron deficiency anemia, thrombocytopenia), renal insufficiency, and anemia 
due to kidney disease. Many factors could lead to the suboptimal thromboprophylaxis observed in this cohort. In addition, a high occurrence of tumors appeared in the elderly patients. Approximately one third of the patients in the over 85 year-old group had tumors in different systems, which affected the function of the blood coagulation system. Furthermore, senile dementia and high risk of falling could increase the difficulty of antithrombotic therapy (Jacobs et al., 2009). In addition, diseases of bleeding or thrombosis appeared in the course of therapy in approximately $50 \%$ of the older patients, and they coexisted in some cases. The factors affecting bleeding and thrombosis were reciprocally interwoven, leading to contradictions of antithrombotic therapy in patients with AF, especially in the elderly. This resulted in contradictions of sufficient antithrombotic treatment, gastrointestinal and brain bleeding, insufficient antithrombosis, and deep vein and atrial thrombosis. However, it was found that patients with a high risk of hemorrhage could obtain a greater benefit from antithrombotic therapy (RobertEbadi et al., 2009). Therefore, elderly patients with AF should carefully assess the risk-benefit ratio of antithrombotic therapy in selecting appropriate treatment strategies.

In conclusion, this data showed several features of elderly patients with AF: 1) various pathophysiologies were observed, including high rates of cancer, which affected blood coagulation, suggesting that the risk-benefit ratio of various treatments needs to be measured to improve the patients' quality of life and extend their lifespans as far as possible; 2) complex pathophysiological statuses were observed in elderly patients with AF, which caused excessive contraindications and relative contraindications, thus limiting the benefits of antithrombotic therapy; 3 ) there were high risks of thrombosis and bleeding in elderly patients, which conflicted with the antithrombotic therapy. The conflict manifested as a higher risk of thrombosis and bleeding complications during treatment, which increased the difficulty to choose antithrombotic therapy and the risk of bleeding in antithrombotic therapy.

Previous risk assessments on hemorrhage during anticoagulation therapy in patients with AF have yielded vague results. The ESC2010 guidelines quantified the bleeding risk assessment by using HAS-BLED programs (Pisters et al., 2010) to evaluate the risk of bleeding in AF patients with anticoagulation therapy. Under these guidelines, a bleeding score of more than 3 points prompted the "high risk" classification, and such "high risk" patients should be cautious when receiving warfarin or aspirin therapy. Two clinical trials, SPORTIF III and SPORTIF IV, showed that the HAS-BLED score could effectively predict the risk of bleeding in patients with AF (Lip et al., 2011). However, whether HAS-BLED programs are suitable for elderly patients with AF in China still needs further confirmation based on clinical evidencebased medicine, especially with respect to the risk stratification of bleeding in senile patients with AF to benefit patients who would receive antithrombotic therapy. Indeed, suboptimal thromboprophylaxis is commonly widely applied in clinical practice, especially in East Asian countries. The rate of oral anticoagulant (OAC) use is $0.5-28 \%$ in China, Singapore, Malaysia, and Taiwan (Guo et al., 2013). A recent Chinese quality evaluation of stroke care and treatment registry demonstrated that OAC was used in only $20 \%$ of 499 stroke patients with $\mathrm{AF}$ (Gao et al., 2013). There are several possible reasons to explain these results. The main reason can be attributed to limitations of warfarin. This drug needs routine monitoring and dose adjustments, and the target internal normalized ratio (INR) range is narrow, which is likely to be influenced by broad interactions with food and other drugs. Secondly, our patients were elderly individuals who were already at risk for thrombotic and bleeding events considering their comorbidities, leading to INR instability and difficulties in regulating INR. Thirdly, the 
risk for bleeding in response to warfarin in Asian populations may be related to differences in the prevalence of certain polymorphisms of genes that influence warfarin pharmacokinetics and pharmacodynamics (Medi et al., 2010). The development of novel oral anticoagulants will provide a valuable therapeutic option in Asian patients with AF who are at risk for stroke.

Only 23 patients were treated with anticoagulation therapy of the elderly patients with $\mathrm{AF}$, leading to a relatively small sample size, which limits the comparison of anticoagulation therapy across different age groups. Therefore, expanding the sample size in a similar study is needed to further confirm whether the 2010 ESC AF guidelines and American ACCF 2011 guidelines can be suitably applied for antithrombotic therapy of "younger" and "older" elderly patients with AF therapy in China.

\section{REFERENCES}

Böhm M, Thoenes M, Neuberger HR, Gräber S, et al. (2009). Atrial fibrillation and heart rate independently correlate to microalbuminuria in hypertensive patients. Eur. Heart J. 30: 1364-1371.

Camm AJ, Kirchhof P, Lip GY, Schotten U, et al. (2010). Guidelines for the management of atrial fibrillation: the Task Force for the Management of Atrial Fibrillation of the European Society of Cardiology (ESC). Eur. Heart J. 31: 2369-2429.

Crandall MA, Bradley DJ, Packer DL and Asirvatham SJ (2009). Contemporary management of atrial fibrillation: update on anticoagulation and invasive management strategies. Mayo Clin. Proc. 84: 643-662.

Freestone B and Lip GYH (2003). Epidemiology and Costs of Cardiac Arrhythmias. In: Cardiac Arrhythmias: A Clinical Approach (Lip GYH and Godtfredsen J, eds.). Mosby, Edinburgh, 3-24.

Fuster V, Ryden LE, Cannom DS, Crijns HJ, et al. (2011). 2011 ACCF/AHA/HRS focused updates incorporated into the ACC/AHA/ESC 2006 guidelines for the management of patients with atrial fibrillation: a report of the American College of Cardiology Foundation/American Heart Association Task Force on practice guidelines. Circulation 123: e269-e367.

Gage BF, Waterman AD, Shannon W, Boechler M, et al. (2001). Validation of clinical classification schemes for predicting stroke: results from the National Registry of Atrial Fibrillation. JAMA 285: 2864-2870.

Gao Q, Fu X, Wei JW, Chen X, et al. (2013). China QUEST Study Investigators. Use of oral anticoagulation among stroke patients with atrial fibrillation in China: the China QUEST (Quality evaluation of stroke care and treatment) registry study. Int. J. Stroke 8: 150-154.

Go AS, Hylek EM, Phillips KA, Chang Y, et al. (2001). Prevalence of diagnosed atrial fibrillation in adults: national implications for rhythm management and stroke prevention: the AnTicoagulation and Risk Factors in Atrial Fibrillation (ATRIA) Study. JAMA 285: 2370-2375.

Guo Y, Pisters R, Apostolakis S, Blann AD, et al. (2013). Stroke risk and suboptimal thromboprophylaxis in Chinese patients with atrial fibrillation: would the novel oral anticoagulants have an impact? Int. J. Cardiol. 168: 515-522.

Jacobs LG, Billett HH, Freeman K, Dinglas C, et al. (2009). Anticoagulation for stroke prevention in elderly patients with atrial fibrillation, including those with falls and/or early-stage dementia: a single-center, retrospective, observational study. Am. J. Geriatr. Pharmacother. 7: 159-166.

Lévy S, Camm AJ, Saksena S, Aliot E, et al. (2003). International consensus on nomenclature and classification of atrial fibrillation; a collaborative project of the Working Group on Arrhythmias and the Working Group on Cardiac Pacing of the European Society of Cardiology and the North American Society of Pacing and Electrophysiology. Europace 5: 119-122.

Lin HJ, Wolf PA, Kelly-Hayes M, Beiser AS, et al. (1996). Stroke severity in atrial fibrillation. The Framingham Study. Stroke 27: 1760-1764.

Lip GY, Nieuwlaat R, Pisters R, Lane DA, et al. (2010). Refining clinical risk stratification for predicting stroke and thromboembolism in atrial fibrillation using a novel risk factor-based approach: the euro heart survey on atrial fibrillation. Chest 137: 263-272.

Lip GY, Frison L, Halperin JL and Lane DA (2011). Comparative validation of a novel risk score for predicting bleeding risk in anticoagulated patients with atrial fibrillation: the HAS-BLED (Hypertension, Abnormal Renal/Liver Function, Stroke, Bleeding History or Predisposition, Labile INR, Elderly, Drugs/Alcohol Concomitantly) score. $J$. Am. Coll. Cardiol. 57: 173-180. 
Marini C, De SF, Sacco S, Russo T, et al. (2005). Contribution of atrial fibrillation to incidence and outcome of ischemic stroke: results from a population-based study. Stroke 36: 1115-1119.

Medi C, Hankey GJ and Freedman SB (2010). Stroke risk and antithrombotic strategies in atrial fibrillation. Stroke 41: 2705-2713.

Pérez-Gómez F, Iriarte JA, Zumalde J, Berjón J, et al. (2007). Antithrombotic therapy in elderly patients with atrial fibrillation: effects and bleeding complications: a stratified analysis of the NASPEAF randomized trial. Eur. Heart J. 28: 996-1003.

Pisters R, Lane DA, Nieuwlaat R, de Vos CB, et al. (2010). A novel user-friendly score (HAS-BLED) to assess 1-year risk of major bleeding in patients with atrial fibrillation: the Euro Heart Survey. Chest 138: 1093-1100.

Robert-Ebadi H, Le GG and Righini M (2009). Use of anticoagulants in elderly patients: practical recommendations. Clin. Interv. Aging 4: 165-177.

Singer DE, Albers GW, Dalen JE, Fang MC, et al. (2008). Antithrombotic therapy in atrial fibrillation: American College of Chest Physicians Evidence-Based Clinical Practice Guidelines (8th edn.). Chest 133: 546S-592S.

Spence JD (2009). Stroke: Atrial fibrillation, stroke prevention therapy and aging. Nat. Rev. Cardiol. 6: 448-450.

Wann LS, Curtis AB, January CT, Ellenbogen KA, et al. (2011a). 2011 ACCF/AHA/HRS focused update on the management of patients with atrial fibrillation (Updating the 2006 Guideline): a report of the American College of Cardiology Foundation/American Heart Association Task Force on Practice Guidelines. J. Am. Coll. Cardiol. 57: 223-242.

Wann LS, Curtis AB, Ellenbogen KA, Estes NA, III, et al. (2011b). 2011 ACCF/AHA/HRS focused update on the management of patients with atrial fibrillation (update on Dabigatran): a report of the American College of Cardiology Foundation/American Heart Association Task Force on practice guidelines. Circulation 123: 1144-1150. 